This page is intentionally left blank 


\section{PROBABILITY THEORY}

\section{Vladimir Rotar}

Central Economic Mathematical Institute Russian Academy of Sciences 


\section{Published by}

World Scientific Publishing Co. Pte. Ltd.

P O Box 128, Farrer Road, Singapore 912805

USA office: Suite 1B, 1060 Main Street, River Edge, NJ 07661

UK office: 57 Shelton Street, Covent Garden, London WC2H 9HE

\section{British Library Cataloguing-in-Publication Data \\ A catalogue record for this book is available from the British Library.}

\section{PROBABILITY THEORY}

Copyright (1) 1997 by World Scientific Publishing Co. Pte. Ltd.

All rights reserved. This book, or parts thereof, may not be reproduced in any form or by any means, electronic or mechanical, including photocopying, recording or any information storage and retrieval system now known or to be invented, without written permission from the Publisher.

For photocopying of material in this volume, please pay a copying fee through the Copyright Clearance Center, Inc., 222 Rosewood Drive, Danvers, MA 01923, USA. In this case permission to photocopy is not required from the publisher.

ISBN 981-02-2213-0

This book is printed on acid-free paper.

Printed in Singapore by Uto-Print 


\section{CONTENTS}

PREFACE TO THE ENGLISH EDITION

xi

INTRODUCTION xiii

0. A space of elementary events xvii

\section{Part I. Elementary Theory}

Chapter 1. Basic Notions

1. Probability distributions on discrete spaces of elementary events

Elementary probabilities. Events and their probabilities.

Properties of probability measures. Summation theorem.

Continuity property. Probability space.

Appendix to Section 1. Particular classical schemes

Permutations. Partitions into groups. Allocations of particles into cells. Random sampling with and without replacement.

2. Independence of events and sequences of trials

Definitions and examples. Bernoulli's scheme. The multinomial scheme.

3. Conditional probability

Definitions and examples. The formula of total probability.

Ruin problem. Bayes' formula.

4. Random variables

One-dimensional random variables. Joint distributions of random variables. Convolutions. Conditional distributions.

5. Expectation

Definitions and examples. Properties of expectation.

Conditional expectations. The formula of total expectation.

Regression. The conditional expectation with respect to a complete set of disjoint events.

6. Variance

Definitions and examples. Two properties of variance. Standard scaling (or normalization). Bienayme's equality. Sums of independent random variables. Arithmetic mean of random variables.

7. Higher moments and inequalities for deviations

Moments. Jensen's inequality. Basic inequality for deviations. 
Chapter 2. The Law of Large Numbers. Limit Theorems for Bernoulli's Scheme

8. The law of large numbers $\quad 50$

Convergence in probability. The weak law of large numbers. Convergence of expectations. Bernstein's polynomials.

Appendix to Section 8

'Zero-sum games. St. Petersburg's paradox. Relative stability. Utility function. The simplest model of insurance.

9. De Moivre-Laplace theorems

The local theorem. The integral theorem. On the accuracy of approximation. Proof of the local theorem.

10. The Poisson theorem and the simplest flow of events

The Poisson theorem. The simplest flow of events. The convolution of Poisson's distributions.

Chapter 3. Generating Functions and Random Walks

11. Generating functions (g.f.'s)

Definition and basic properties. The generating function of the sum of random variables. The generating function of the sum of a random number of random variables. The extinction problem or the Galton-Watson process. The generating function of a convolution. Continuity theorem. The Poisson theorem for nonidentically distributed summands.

12. On random walks

"Return to zero". The arcsine law. The number of "draws".

\section{Part II. General Theory}

Chapter 1. Foundations of the Theory

1. Preliminary constructions

Probability measure. Random variables and their expectations.

2. Probability distributions

Events. Measures and probabilities. Continuity property.

Products of probability spaces.

3. Random variables

Measurability. Convergence of random variables.

The Lebesgue integral or expectation of an elementary random variable. The Lebesgue integral: expectation of an arbitrary 
random variable. The integral over a set and the restriction of a measure. Passage to the limit under the integral sign. Comparing the Riemann and Lebesgue integrals. Integrals with respect to $\sigma$-finite measures. The theorem on iterated integrals. Absolute continuity and the Radon-Nikodym theory. Concluding remarks.

\section{Chapter 2. Distributions on Finite-Dimensional Spaces}

4. Distributions of random variables

Induced distributions. Absolutely continuous distributions.

Discrete distributions. Mixtures. Distribution functions

(d.f.'s). Singular distributions. The Lebesgue expansion.

Lebesgue-Stieltjes integral. Linear transforms of random variables and types of distributions.

5. Distributions of random vectors

Definitions and examples. Linear transforms of random vectors. Independent random variables. Convolutions.

6. Some particular distributions on the real line

The uniform type. The normal type. The exponential type and the "lack of memory" property. Gamma-distributions. A connection between the Poisson and exponential distributions. $\chi^{2}$-distribution.

7. Covariance analysis and multivariate normal distribution

Covariance. Covariance matrix. Properties of covariance matrices. The method of principal components or main factors. Standard normal distribution in $\mathbf{R}^{k}$. Normal distribution in $\mathbf{R}^{k}$.

8. Convergence of distributions

Weak or proper convergence. Convergence of moments.

Stochastic boundedness and the selection theorem. Improper convergence. Proximity of distributions. Other types of convergence of distributions. Multidimensional case. Proofs of Theorems 1-1' and Lemma 4. Proof of Theorem 2. Proofs of Theorems $3-3^{\prime}$. Proofs of Theorems 4-5.

9. Comparison of distributions

Preference relation or ordering. Calculability. Primary conditions. Comparison based on expectation and variance. Continuity conditions. Expectation as a criterion. Utility function. Utility function and Condition A1. 
Jensen's inequality. Utility function and Condition A2.

Generalization of Theorem 2. Allais' paradox and nonlinear functionals.

\section{Chapter 3. Conditional Distributions}

10. Conditional distributions and expectations

The discrete case. The absolutely continuous case. The general case. Properties of conditional expectation. The formula of total expectation. A regression function and a prediction problem. Linear regression. The convolution formula. Product of independent random variables. Ratio of independent random variables. A system of equations with random coefficients. The Student distribution.

11. Conditional expectations with respect to $\sigma$-algebras of events

Basic notions. Properties of conditional expectation. Conditional distributions. Derivation of representation (5) from Section 10.

Chapter 4. Some Kinds of Dependence

12. Conditionally independent and exchangeable random variables

Mixtures of distributions. Conditionally independent random variables. Symmetrically dependent or exchangeable random variables.

13. Martingales

Definitions, properties and examples. Markov moments and boundary problems. Continuity properties. Proof of De Finetti theorem.

14. Markov chains

Basic notions and properties. Ergodicity property.

Chapter 5. Limit Theorems

15. Limit theorems for maxima and minima. Regularly varying functions

The statement of the problem. Preliminary result.

Regularly varying functions. Limit theorems for identically distributed random variables. Proofs of Theorems 1-2. The general scheme. 


\section{Characteristic functions and the first limit theorems}

for sums

The Laplace transform. Elementary properties of characteristic functions. Examples of characteristic functions. Characteristic functions of lattice distributions. The characteristic function of a mixture of distributions. Uniqueness and continuity theorems. On smoothness of characteristic functions. The law of large numbers for identically distributed summands. Convergence of sums of identically distributed summands to a normal distribution: Lévy's theorem. A limit theorem for sums of rotations.

17. Characteristic functions, inversion theorems

Lattice distributions. Absolutely continuous distributions.

The inverse in the general case: smoothing. An inversion formula based on the Dirichlet integral. Truncation inequalities. Proofs of the continuity theorems. Generalization of the continuity theorem.

18. Limit theorems for sums. The case of finite variances

Preliminary statement of the problem. Triangular arrays.

Conditions for normal convergence: sufficiency. Conditions for normal convergence: necessity. Convergence to the Poisson distribution. The theorem on proximity of convolutions. Lindeberg's theorem follows from Theorem 5. Proof of Theorem 5. Proof of Feller's theorem. Development of Theorem 4 from Theorem 5 .

\section{Stable distributions}

Stability property. The stability of limiting distributions. The case of finite variances. Symmetric stable distributions. On the density of symmetric stable distributions. Stable distributions in the general case. Convergence to stable distributions. Proof of the theorem on the number of draws. Additional remarks.

20. Limit theorems for sums. The general case

Centering. Rescaling. The general scheme of summation. Proof of Theorem 1. Convergence to stable distributions.

\section{On large deviations}

The statement of the problem. A theorem on large deviations. An inequality for large deviations. Proof of Theorem 2. 
22. Characteristic functions and limit theorems in the multidimensional case

Characteristic functions. Uniqueness and continuity theorems.

The multidimensional analog of Lévy's theorem.

Limit theorems for some nonlinear functions of many random arguments.

23. Limit theorems for dependent summands

Preliminary examples. Random summands with two values. Limit theorems for an infinite sequence of symmetrically dependent random variables. Limit theorems for martingales. The derivation of Theorem 6 from Theorem 7. Proof of Theorem 7. Limit theorems for random variables defined on a Markov chain.

24. Nonlinear limit theorems

The general statement of the problem. Multilinear forms and polynomials of random variables.

\section{Chapter 6. Almost Sure Behavior of Sums of Random}

Variables

25. The Borell-Cantelli theorem and the "zero-one" law

The Borell-Cantelli theorem. The "zero-one" law.

26. The strong law of large numbers (SLLN) and the law of the iterated logarithm

The strong law of large numbers: necessity. The Kolmogorov and Lévy inequalities. A theorem on convergence of martingales or a criterion of convergence of series. The strong law of large numbers: sufficiency. The strong law of large numbers for independent identically distributed random variables. The law of the iterated logarithm.

27. The law of large numbers and stochastic optimization problems

The simplest stochastic optimization scheme. The case of dependent observations. On the general scheme of stochastic optimization. 


\section{PREFACE TO THE ENGLISH EDITION}

This text may be useful for students who are trained in Probability Theory, however it is intended for a larger audience. Specifically, the book is written for people who are going to use Probability Theory mostly in applications, but are willing to do so at a highly professional level. In view of this the exposition is rigorous and proofs are, with very rare exceptions, complete. On the other hand, much attention is being paid to the interpretation and the "physical" sense of results, examples and possibilities of applications. Some material which is interesting only from a purely mathematical point of view is omitted and, on the other hand, some topics which are important for possible applications are added.

The prerequisite is good calculus. Throughout the entire book, additional remarks and the parts of proofs, which are purely technical in character and may be omitted without essential losses in understanding, are given in special reduced fonts in order to facilitate reading.

The basic necessary material - that is to say, an introductory course - is given in Sections 1-11 of Part I and Sections 1-10, 12-18, 22, 25-26 of Part II. The exposition of this material is self-contained.

The rest of the book is for deeper study and may be of help for teachers and scientists in Probability and Statistics as a reference and for further development of complementary or special courses.

It is impossible to avoid subjectivity entirely in selecting material for a text on such a subject as Probability, a theory well-developed and frequently emerged from applications. In this book a rather broad spectrum of topics is involved, however the strongest focus is on various limit theorems, linear and non-linear. These results seem to be the deepest and most significant at least among those which can reasonably be presented in an introductory course.

The book contains also material which is not traditional for texts on the theory of probability, but is topical in light of modern requirements for applications. Two 
cases in point are risk evaluation theory (that is, the theory of orderings in spaces of probability distributions) and stochastic optimization.

Regarding some topics, I have been trying to take into account some recent achievements of the theory. This is concerned not only with new results - for example, limit theorems for sums of dependent variables (e.g., martingales or exchangeable variables), non-linear and non-classical limit theorems - but also with unified approaches to the exposition of well-known facts and available techniques which may be of help in simplifying the proofs.

Acknowledgements. First of all I should record my fondest thanks to my dear teacher Academician Yu. V. Prokhorov from whom I learned Probability Theory itself, how to work in it as well as how to teach it.

This text is based partially on the course which I taught at Moscow University MIEM in the Department of Probability and Statistics. I enjoyed the help and collaboration of all members of this department.

I am truly grateful to Professors V. M. Kruglov, Ya. Yu. Nikitin and E. L. Presman whose comments and criticism led to corrections and improvements.

In preparing the English version of the book I benefited from comments of Professors I. Abramson, P. Fitzsimmons, L. Haff and Y. Rinott, for which I am very thankful.

Vladimir Rotar

December, 1996

Moscow - San Diego 


\section{INTRODUCTION}

Probability Theory is concerned with modelling phenomena whose nature involves elements of uncertainty. In other words, Probability Theory deals with models of experiments whose outcomes cannot be precisely predicted. Tossing a coin, the weather tomorrow, the duration of the uninterrupted operation of a machine, the proportion of defective articles in a large batch of items, noises under transmission of an electric signal and more fall into the category of phenomena which can be studied by means of probabilistic methods.

It is worth noting, however, that the conclusions of this theory, at least of its classical part, which is the object of investigation in the present textbook, applies well to real phenomena possessing the so-called stochastic stability property. This property means that the experiments concerned can be, at least in principle, repeated indefinitely often under the same conditions. As to one-of-a-kind experiments, one can hardly study them by means of probabilistic constructions.

The description given above is by no means a definition of stochastic stability. The best way of understanding the essence of this concept is to consider the fundamental notions of the theory itself and, first of all, the so-called law of large numbers. In this introduction we restrict ourselves to the following comments.

In our minds the probability or the "degree of possibility" of an event is related to the frequency of the occurrence under a large number of repetitions of homogeneous trials. One classical and much applicable example, "fair" tosses of an "unbiased" coin can add interest and aid in understanding. Let $n$ be the number of tosses and $m_{n}$ be the fraction of tosses in which "heads" have appeared. Intuitively one might expect (and experiments confirm this) that if tossing is performed in a way so that the results of separate tosses would be independent, then, for large $n$, the quantity $m_{n}$ should be close to $\frac{1}{2}$, meaning

$$
m_{n} \longrightarrow \frac{1}{2} \quad \text { as } n \longrightarrow \infty
$$


The conclusion can be drawn from this observation that the probability of a "head" appearing in a particular trial may be taken to be $\frac{1}{2}$.

The basic difficulty, however, is that it is impossible to give a rigorous meaning to these arguments within standard calculus. First, it is not clear how to define rigorously the concept of independence. Second, the quantity $m_{n}$ is random, it being understood that its values differ for different series of tosses. For example, it may happen that a "head" appears in each toss, thus causing $m_{n}=1$. This means that we are unable to explain the relation $(*)$ with the aid of the definition of limit of numerical sequences since $m_{n}$ is a "random variable" rather than an ordinary number.

In trying to overcome this deficiency it is fairly common in the contemporary science to construct a rigorous axiomatic model of "random phenomena". Such a model necessitates imposing simple rules for calculating probabilities and formulating the definitions of independency and convergence of random variables. It is this subject we discuss at the beginning of the theory.

With respect to tossing a coin, the model we have mentioned above leads to the following. Under the a priori premise that probability can be measured by a numerical value and that the probability of the occurrence of a "head" in a sequence of independent tosses is equal to $\frac{1}{2}$ for each trial, one can calculate the probability of the rather "complicated" event that the quantity $m_{n}$ deviates from $\frac{1}{2}$ by a value exceeding a number $\varepsilon$, which is kept fixed. We denote this probability by $p_{n}(\varepsilon)$. A particular instance of the law of large numbers is that for any arbitrarily small $\varepsilon>0$

$$
p_{n}(\varepsilon) \longrightarrow 0 \text { as } n \longrightarrow \infty,
$$

thereby clarifying the meaning of relation $(*)$.

Similar results are still valid in a more general situation: the fraction of the occurrence of some event under multiple repetitions of an experiment is indeed close to the formally defined probability of the event if the notion of closeness is to be understood in the sense of $(* *)$.

Relations of the kind (*) (or $(* *)$ ) do justify the model under discussion: they are not only consistent with real-life situations and approve the frequency interpretation of the notion of probability, but also enrich intuitive ideas.

The simplest version of the law of large numbers owes a debt to James Bernoulli (1654-1705). His paper "Ars Conjectandi" ("The Art of Guessing") published in 1713 can be regarded as an origin of Probability Theory although a number of 
notions and problems (related mainly to calculations of probabilities in games of chance) have been under consideration earlier by B. Pascal (1623-1662), P. Fermat (1601-1665), C. Huygens (1629-1695) and others.

Much progress in a number of limit theorems of Probability Theory has been achieved by A. De Moivre (1667-1754), P. Laplace (1749-1827), S. Poisson (1781$1840)$ and K. Gauss (1777-1855). Their results have not lost their significance even today.

A very fruitful period is connected with the names of P.L. Chebyshev (18211894), A.A. Markov (1856-1922) and A.M. Lyapunov (1857-1918) who provided not only new statements of problems, notions and theorems, but also powerful methods for solving many problems which do arise in this branch of science. Further development of Probability Theory is stipulated by constructing its axiomatics. The first works in this direction are due to E. Borel (1871-1956), S.N. Bernstein (1880-1968) and R. von Mises (1883-1956) whose key results are available in the present textbook. The ultimate solution to the axiomatization problem came with A.N. Kolmogorov (1903-1987). His celebrated "Foundations of Probability Theory" provides sufficient background for this theory. The rapid development in the last half-century led to a rich and diverse mathematical discipline.

Several words about the structure of the book. The exposition starts with Section 0 in which the initial notion of the space of elementary events is introduced. The remaining material is presented in two parts: "Elementary Theory" (Part I) and "General Theory" (Part II). Each part is divided into chapters, sections and subsections. The numeration of sections within each part is self-contained. We follow the same principle when numbering theorems, definitions, examples, and so on, within a section. The approved system of references is clear from the following examples: Subsection 4 is the fourth subsection in a current section; Subsection 2.4 is the fourth subsection in Section 2; Lemma 3 is the third lemma in a current section; Lemma 3.5 is the fifth lemma of Section 3 from a current part, and so on. The symbol $\square$ refers to either the end of the proof of a statement or the end of considering an example. As we have mentioned in Preface, the material which may be omitted at the first reading is placed in the reduced fonts between the symbols $\boldsymbol{\nabla}$ and $\boldsymbol{\Lambda}$. All exercises are enclosed into frames. 
Throughout the entire book, we use the following abbreviations:

a.s. - almost surely;

a.e. - almost everywhere;

EE - elementary events;

SEE - space of elementary events;

r.v. - random variable;

r.vec. - random vector;

ch.f. - characteristic function;

d.f. - distribution function;

g.f. - generating function;

i.i.d. - independent identically distributed;

LLN - the law of large numbers;

SLLN - the strong law of large numbers;

CLT - central limit theorem. 


\section{A space of elementary events}

Space of elementary events (SEE) or space of elementary outcomes is a primitive notion. Formally a SEE is a set of elements of arbitrary nature. We will regard it as the set of the "smallest", indecomposable, disjoint events that may occur as a result of an experiment under study. We use the symbol $\Omega$ to denote a SEE and the symbol $\omega$ for its elements. Thus, $\Omega=\{\omega\}$. The cardinality of $\Omega$, that is, the number of its elements is written as $|\Omega|$.

\section{Examples.}

1. A toss of a coin. Two elementary events are possible: $\omega_{1}$ is the event that the "head" appears and $\omega_{2}$ is the event that the "tail" appears. Here we exclude the possibility that the coin stands on edge. Thus, $\Omega=\left\{\omega_{1}, \omega_{2}\right\}$ and $|\Omega|=2$.

2. A toss of a die with six faces numbered from 1 to 6 . Here $\Omega=\left\{\omega_{1}, \ldots, \Omega_{6}\right\}$, where $\omega_{i}$ corresponds to the occurrence of the number $i$ on the top face.

3. Two tosses of a coin. Here one can designate elementary events as $\mathrm{HH}$ (the "head" appears twice), HT (at the first toss the "head" appears and at the second the "tail" appears), TH and TT. Clearly, $|\Omega|=4$. It is worth emphasizing that the outcomes $\mathrm{HT}$ and $\mathrm{TH}$ are different.

4. A sequence of trials or experiments in each of which an event of interest may occur. We will speak about a "success" if the event occurs and about a "failure" if it fails to hold. For example, while tossing a coin we may label successful the tosses at which "heads" appear. Considering the birth of children, we may think about a "success" if a girl is born. In checking a batch of items, a success consists of fitting an item to an approved standard. Let $n$ be the number of trials. We label by 1 a "success" and by 0 a "failure" whose use permits us to identify each elementary event with a sequence of zeroes and units. For example, the expression $\omega=1001$ means that $n=4$ and the first and fourth trials (and they only) were successful. The number of all elementary events is $|\Omega|=2^{n}$. This is due to the fact that each elementary event is connected with the binary notation of a number. There are $2^{n}$ different numbers of this kind (including 0 ) having no more than $n$ digits.

The SEE's we have mentioned above are finite. 
5. Claims in a queuing system. The claims and the system itself can be understood in a broad sense. Cases in point are telephone calls to a telephone exchange, customers in a supermarket, information signals entering a block of a computer, cosmic rays recorded by a detector, and so on. If we are only interested in the number of claims coming to a system per unit time it suffices to consider $\Omega=\left\{\omega_{0}, \omega_{1}, \ldots\right\}$, where $\omega_{i}$ is the elementary event reflecting the appearance exactly $i$ claims. Although the total number of claims cannot be as large as we like, it is reasonable to accept $|\Omega|=\infty$. The SEE under consideration is countable.

6. Waiting time. If you use trains in a subway at random time moments and interarrival times are all one unit of time, you can identify each elementary outcome with a number $x$ from the segment $[0,1]$ and $\Omega$ with the segment itself.

7. The error of an observation may be positive or negative. In this view, it is natural to adopt the real line as $\Omega$.

8. A flow of events. Let us return to Example 5 and consider the process of claims appearing in time. In this case one can speak about a flow of events by regarding the appearance of a claim as an event. Clearly, each realization of the flow is uniquely determined by the times at which the claims appear, making it possible to identify the SEE with $R^{\infty}$, the space of all infinite numerical sequences.

Of course, one can point out more complex SEE's: observation over the time evolution of the value of electric current leads to a functional SEE, where each elementary event is a function, observation over an ocean wave leads to a SEE where each elementary event is a surface, and so on. Any SEE consisting of a finite or a countable number of $\mathrm{EE}$ is said to be discrete, while others are called uncountable.

9. A sequence of infinite tosses of a coin. One can identify each elementary event with an infinite zero-one sequence. The SEE so constructed is uncountable. Indeed, let us add the symbols 0 and, at the beginning of each such sequence. As a result we get the binary notation of a number lying within the segment $[0,1]$.

Rigorous constructions of Probability Theory for the general SEE cause certain difficulties which one can avoid for the discrete case while preserving the ideas underlying many results. For this reason our exposition begins with the theory of discrete SEE's ( Elementary Theory); we consider in passing some problems formally connected with uncountable SEE's but the solutions of which can be found by means of discrete methods. 